\title{
38. Prise en charge des AVC du sujet âgé
}

\author{
(c) Springer-Verlag France 2010
}

\author{
38.2

Quelle est la prise en charge des crises d'épilepsies
au cours et au décours des AVC?
J.-L. de Reuck
Département de neurologie, hôpital universitaire, Gand, Belgique

Malgré que des directives générales existent concernant le diagnostique et le traitement de l'épilepsie, il n'y a actuellement pas de recommandations spécifiques concernant la prise en charge des crises épileptiques en relation à un $\mathrm{AVC}$.

L'incidence des crises épileptiques après un AVC a été estimée à $8,9 \%$ dans la seule étude prospective. Il s'agit probablement d'une sous-estimation vu que les crises au cours de l'AVC et les accès non convulsifs sont rarement reconnues comme telle.

Les crises focales simples sont de loin les plus fréquentes. Les crises sans convulsions et l'état de mal représentent chacun à peu près $10 \%$ et apparaissent le plus fréquemment au cours de l'AVC.

Les malades avec un déficit neurologique partiel et un infarctus cortical au niveau des lobes pariétal et temporal sont le plus à risque de développer des crises d'épilepsies tardives. Les AVC d'origine cardioembolique peuvent récidiver et se présenter comme une crise d'épilepsie tardive.

Les facteurs de risque, le diagnostique, la prise en charge et le traitement seront différents selon le temps d'apparition des crises épileptiques en relation à l'AVC.

$\mathrm{Vu}$ le fait, d'une part, que les crises répétitives et l'état de mal aggravent la condition neurologique et mentale des malades avec AVC, et que, d'autre part, la plus part des antiépileptiques aggravent le déficit cognitif, il est important de sélectionner quels malades avec un AVC doivent être traités avec des antiépileptiques et quel est le médicament de choix.

Les malades avec crises au cours d'un AVC ne développent que dans $10 \%$ des cas des crises tardives. Un traitement préventif n'est donc pas souhaitable. Les crises apparaissant dans les mois après l'AVC doivent être traités après le premier accès car les récidives sont très fréquentes $(55 \%)$ et aggravent le déficit neuropsychiatrique. Les crises apparaissant très tardivement après l'AVC ont un pronostique plus favorable. Le traitement par antiépileptiques est uniquement nécessaire en cas de récidives $(40 \%)$.

Malgré que la carbamazepine reste le premier choix, les nouveaux antiépileptiques comme la lamotrigine, la gabapentine et le leviteracetam sont des alternatives en cas d'effets secondaires ou de control insuffisant des crises.
38.4

Symposium AVC du sujet âgé : quel est le risque de démence en post-AVC ? D. Zekry

Département de réhabilitation et gériatrie, hôpital des Trois-Chêne, Thônex, Suisse

Une démence post-AVC est rapportée dans près d'un tiers des cas. Non seulement les AVC sont un facteur de risque de démence, mais la démence est un facteur de risque d'AVC. Un état démentiel préexistant à la survenue d'un AVC est retrouvé dans en moyenne $15 \%$ des cas. Or la démence (qui est le plus souvent de nature dégénérative de type maladie d'Alzheimer) n'est pas diagnostiquée dans la plupart des cas au moment de l'AVC. L'incidence des AVC et celle de la démence sont liées chez les personnes de plus de 85 ans. Les facteurs qui expliquent l'apparition d'une démence à la suite d'un AVC sont nombreux. Il est pratique, pour le neuropathologiste comme pour le clinicien, de distinguer les démences dues à des lésions de grande taille (infarctus, le plus souvent) et celles qui relèvent de lésions de petite taille (lacunes) ou qui sont diffuses (encéphalopathies sous-corticales). Concernant les lésions de la macrocirculation, on peut citer la bilatéralité ou le caractère diffus des lésions ; le volume important des lésions ; la topographie du tissu détruit ; l'atteinte, parfois très focale, des systèmes qui règlent la mémoire, l'attention, le raisonnement (circuits limbique ou amygdalothalamofrontal, afférences frontales d'origine sous-corticale), la lésion directe du lobe frontal qui contrôle la programmation et la surveillance des activités mentales ainsi que la lésion des aires associatives. Des lésions de faible volume situées dans des sites stratégiques sont aussi susceptibles de créer un affaiblissement intellectuel ou d'y contribuer. Concernant la microcirculation, les lésions diffuses de la substance blanche, observées dans les leucoencéphalopathies, notamment dans la maladie de Binswanger associée à l'hypertension artérielle, peuvent provoquer une démence. Deux études récentes ont montré le rôle des lésions ischémiques microscopiques corticales et sous-corticales chez des personnes âgées. La première a relevé que la démyélinisation périventriculaire expliquait $10 \%$ de la variance du fonctionnement cognitif, la démyélinisation diffuse $4,6 \%$ et les micro-infarcts corticaux $36 \%$. La deuxième a trouvé que les lacunes thalamiques et des noyaux gris centraux avaient un impact significatif sur la cognition ; au contraire, les lacunes de la substance blanche dans les régions frontales, temporales et pariétales ne semblaient pas avoir d'effet important tant qu'elles étaient en nombre relativement faible. Pourtant, il faut signaler qu'il est rare qu'un trouble mnésique soit absolument pur, qu'une démence soit seulement corticale ou sous-corticale. De nombreuses structures, souvent associées, sont ainsi impliquées dans les processus divers menant à la démence. Il est bien plus fréquent que l'on ne puisse dissocier, ni même privilégier, l'un ou l'autre de ces mécanismes. Les progrès thérapeutiques dans la lutte contre la démence et les déficits cognitifs graves chez les patients ayant souffert d'un AVC seront aussi discutés dans ce symposium. 